\title{
Proton beam radiotherapy for esophagus cancer: state of the art
}

\author{
Kimberly R. Gergelis ${ }^{1}$, Krishan R. Jethwa ${ }^{2}$, Erik J. Tryggestad ${ }^{1}$, Jonathan B. Ashman ${ }^{3}$, \\ Michael G. Haddock ${ }^{1}$, Christopher L. Hallemeier ${ }^{1}$
}

${ }^{1}$ Department of Radiation Oncology, Mayo Clinic, Rochester, MN, USA; ${ }^{2}$ Department of Therapeutic Radiology, Yale University School of Medicine, New Haven, CT, USA; ${ }^{3}$ Department of Radiation Oncology, Mayo Clinic, Phoenix/Scottsdale, AZ, USA

Contributions: (I) Conception and design: All authors; (II) Administrative support: None; (III) Provision of study materials or patients: None; (IV) Collection and assembly of data: None; (V) Data analysis and interpretation: None; (VI) Manuscript writing: All authors; (VII) Final approval of manuscript: All authors.

Correspondence to: Christopher L. Hallemeier, MD. Associate Professor in Radiation Oncology, Department of Radiation Oncology, Mayo Clinic, Rochester, MN, USA. Email: Hallemeier.Christopher@mayo.edu.

\begin{abstract}
The majority of esophageal cancer patients are diagnosed with locoregionally confined disease, which is often amenable to curative intent therapy. Chemoradiotherapy (CRT) improves overall survival (OS) in stage II and III esophagus cancer in the neoadjuvant and definitive settings. Due to the close proximity of organs at risk (OARs), including lungs, heart, stomach, bowel, kidneys, and spinal cord, esophageal CRT can result in profound acute and late toxicities. Acute toxicities can include esophagitis, nausea, vomiting, fatigue, and cytopenias. Late complications may also occur months or years after completion of thoracic radiotherapy, including significant cardiac, pulmonary, liver, kidney, or bowel toxicities, which can be life-threatening or fatal. Photon-based radiotherapy exposes OARs to significant doses of radiation, whereas proton beam therapy (PBT) has unique physical properties, as it lacks an exit dose. This allows PBT to deliver, a more conformal dose to the target and minimize the volume of OARs exposed to radiation. This dosimetric advantage may portend an increased therapeutic ratio of CRT for esophagus cancer. The objective of this review is to discuss the evolution of photon and proton-based radiotherapy techniques, rationale, dosimetric and clinical studies comparing outcomes of photon- and proton-based techniques, ongoing prospective trials, and future directions of PBT as a means of reducing toxicity and improving oncologic outcomes for patients with esophagus cancer.
\end{abstract}

Keywords: Esophagus cancer; chemoradiation; proton beam therapy (PBT)

Submitted Apr 01, 2020. Accepted for publication May 04, 2020.

doi: 10.21037/jtd-2019-cptn-06

View this article at: http://dx.doi.org/10.21037/jtd-2019-cptn-06

\section{Introduction}

Esophagus cancer is an uncommon, yet aggressive, malignancy with an estimated 18,440 new cases to be diagnosed and 16,170 deaths attributable to esophagus cancer in the United States in 2020 (1). The majority of patients are diagnosed with locoregionally confined disease and may be amenable to curative intent therapy. The survival rate for patients with esophagus cancer is poor, with only $20 \%$ of all patients surviving 5 years; however, those with localized and locoregional cancer have 5 -year survival rates of $47 \%$ and $25 \%$, respectively (2).

The standard of care for patients with stage II-III thoracic esophagus cancer includes neoadjuvant external beam radiotherapy with concurrent chemotherapy (chemoradiotherapy, CRT) followed by esophagectomy (trimodality therapy), or definitive CRT (without esophagectomy). Gastroesophageal junction cancers can also be treated with this paradigm, although sometimes are treated with perioperative chemotherapy. Cancers of the cervical esophagus are often treated non-operatively with definitive CRT, as surgical removal of these lesions may 


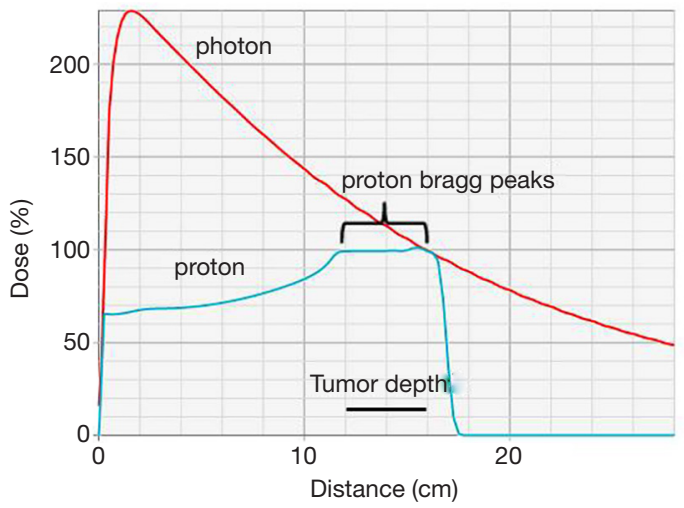

Figure 1 Depth-dose curves for a single photon (red) or proton (blue) beam treating a $4 \mathrm{~cm}$ tumor at 12 to $16 \mathrm{~cm}$ depth. Note greater dose deposited proximal (entrance) and distal (exit) to the tumor with the photon versus proton beam.

require laryngopharyngectomy with a permanent tracheal stoma. Preoperative CRT is utilized to improve overall survival (OS), compared to surgical management alone (3).

Despite the advancements in photon radiotherapy, there continue to be significant acute adverse effects which occur during CRT for esophageal cancer, including esophagitis, nausea, vomiting, fatigue, and cytopenias. Patients may experience significant cardiac, pulmonary, liver, kidney, or bowel toxicities which may occur months or years after completion of thoracic radiotherapy and can be life-threatening or fatal even in the absence of cancer recurrence (4-7). For patients who undergo subsequent surgery, there are potential postoperative pulmonary, cardiac, and gastrointestinal complications (8). Each of these adverse effects may be attributed in part to the radiation dose-volume effects upon normal tissues adjacent to the esophagus in the central thorax or upper abdomen, including the heart, lungs, liver, kidneys, and bowel (9-11). Radiation dose to the heart has been associated with cardiac morbidity and mortality in thoracic malignancies, such as lung and breast $(12,13)$. Novel treatment strategies, such as advanced and highly conformal radiotherapy utilizing proton bream radiotherapy, may offer one such treatment strategy to mitigate toxicity and improve the clinical outcomes for these patients.

The purpose of this review is to discuss the evolution of radiotherapy techniques and the emerging utility of proton beam radiotherapy (PBT) as a means of reducing toxicity and improving oncologic outcomes for patients with esophagus cancer.

\section{Photon radiotherapy techniques}

Radiotherapy was traditionally planned using twodimensional techniques (2D-RT) with large treatment fields to ensure target coverage despite a relative lack of accurate target delineation or an ability to account for most sources of inter- and intra-fraction target variability. Treatment was delivered with megavoltage photons which are associated with substantial entrance and exit dose. Considering the central location of the esophagus, these techniques were associated with significant radiation exposure to adjacent vital organs, including the heart, lungs, spinal cord, liver, kidneys, and bowel.

The advent of computed tomography (CT)-based three-dimensional conformal radiation therapy (3D-CRT) allowed enhanced target and normal anatomy delineation and enabled dose-volume histogram (DVH) reporting. The DVH characterizes the volume of organs at risk (OARs) exposed to various radiation doses. DVHs have been utilized to associate dose-volume parameters with oncologic outcomes as well as radiation-related morbidity and mortality (14).

The subsequent development of intensity modulated radiotherapy (IMRT), which utilizes photon beams at many different angles and has the ability to modify the intensity of the beam at different points in the treatment field, yielded even more conformal treatment plans and a reduction of normal tissue exposure to high doses of radiation when compared to 3D-CRT. Retrospective data suggest that patients with esophageal cancer treated with IMRT have better survival compared to 3D-CRT, possibly due to reduction in non-cancer related deaths, specifically cardiac death (15-17). However, a commonality of each of these techniques is the utilization of mega-voltage photons which have substantial entrance and exit dose through vital organs adjacent to the esophagus.

\section{Physical properties of proton beam therapy}

PBT has further improved the conformality of radiation therapy (Figure 1). A proton beam travels through tissue with low dose deposition along its entrance path. However, as the particles slow down, the energy deposition of PBT rises rapidly and results in most of the energy being deposited at the end of the ionization track with little or no 
exit dose. This peak of the deposition of absorbed energy at a specified depth is known as the Bragg peak $(18,19)$.

When proton beams are produced by an accelerator, they are very small, measuring only a few millimeters in diameter. There are currently two main methods of widening the proton beam used in clinical systems: passive scattering and active scanning. Passive scatter proton therapy (PSPT) was developed first and spreads the beam over depth using a range modulating wheel and laterally using a scattering foil. After the beams are scattered, they are then shaped using collimators with beam-specific apertures or multileaf collimators. The passive scatter method spreads out the Bragg peak, which leads to an increase in the entrance dose proximal to the target. Active scanning, also known as pencil beam scanning (PBS) or intensity modulated proton therapy (IMPT), is a more recent development which utilizes magnets to steer the proton beam, and the energy of the beam is varied in order to shift the Bragg peak position in the $\mathrm{x}, \mathrm{y}$, and $\mathrm{z}$ dimensions (18). IMPT is inherently more conformal than PSPT, delivering lower dose to adjacent tissues.

\section{Dosimetric advantages of proton beam therapy}

Treatment with protons has a dosimetric advantage over photons, as photons deliver peak dose near their entrance into the tissue and have significant exit dose, as opposed to protons, which deliver the peak dose in the target and have little to no exit dose (Figure 1) (19).

Compared with IMRT, PBT for esophagus cancer is capable of delivering the same RT dose to the target volume while delivering lower radiation doses to OARs (Figure 2). A study comparing radiation exposure to the heart and cardiac substructures of patients treated with IMRT versus PBT for mid- to distal esophagus cancer found that treatment with PBT resulted in significantly lower mean heart dose, as well as lower heart volumes exposed to 5-40 Gy (20). There was also lower radiation exposure to several cardiac substructures. It was observed that those treated with IMPT had lower mean heart doses when compared to PSPT. Zhang et al. performed a study comparing normal tissue sparing of PSPT and IMRT for esophageal cancer and demonstrated that lung volumes exposed to 5-20 Gy and mean lung dose were significantly lower with two- or three-beam PSPT (21).

As the majority of treatment failures after definitive CRT for esophageal cancer occur within the gross tumor volume, dose escalation has been postulated as a potential method of increasing local control. A study by MD Anderson Cancer
Center compared dosimetric parameters to see if utilizing IMPT led to reductions in lung and cardiac doses in the setting of dose escalation to $65.8 \mathrm{~Gy}$ in 28 fractions to the gross tumor volume for unresectable distal esophageal cancer (22). Using a 3 field approach (anteroposterior, left posterior oblique, and right posterior oblique beams), IMPT resulted in lower heart and lung doses, compared with IMRT. PBT may have the capability to deliver escalated doses to the distal esophagus, while sparing the nearby OARs; however, the efficacy and safety of dose escalation warrants prospective investigation.

\section{Proton treatment planning}

The physical dose deposition properties of proton beams allow for conformal delivery of radiotherapy dose to the target in "ideal conditions." However, there is increased sensitivity of the dose distribution (relative to photon plans) to inter- and intra-fractional changes in patient anatomy (23). Small changes in tissue density in the beam path (water equivalent thickness, WET) can alter the radiological path length, resulting in under-coverage of tumor and/or excess exposure of normal tissue to radiation. Examples of inter-fractional anatomic changes include patient setup variation, patient weight changes, tumor size and position changes, lung density changes, presence or absence of an esophageal stent or nasogastric feeding tube, and gastric filling or air variation. Intra-fractional anatomic changes, which are predominately driven by respiratory and cardiac motion, can lead to a heterogeneous dose distribution due to the associated "interplay effect." Additionally, diaphragmatic movement during respiration can result in changes in the WET traversed by the beam, resulting in additional interplay effects (24). In order to capture this motion, it is important to obtain a respiratory-correlated fourdimensional treatment CT-simulation scan. For tumors of the distal esophagus and gastroesophageal junction, respiratory motion can be on the order of $2 \mathrm{~cm}$ in the superior-inferior direction (25). In this context, various respiratory motion management strategies can be utilized to minimize dose heterogeneities, including abdominal compression and beam gating (breath hold or phase- or amplitude-based gating). Furthermore, repainting strategies can improve dose homogeneity. Repainting is a method of delivery redundancy, which is achieved by the PBS multiple times over a moving target in order to decrease the interplay effect of tumor motion by statistical averaging. Robust treatment planning is recommended in an attempt to mitigate potential interand intra-fractional uncertainties (23). Careful selection 

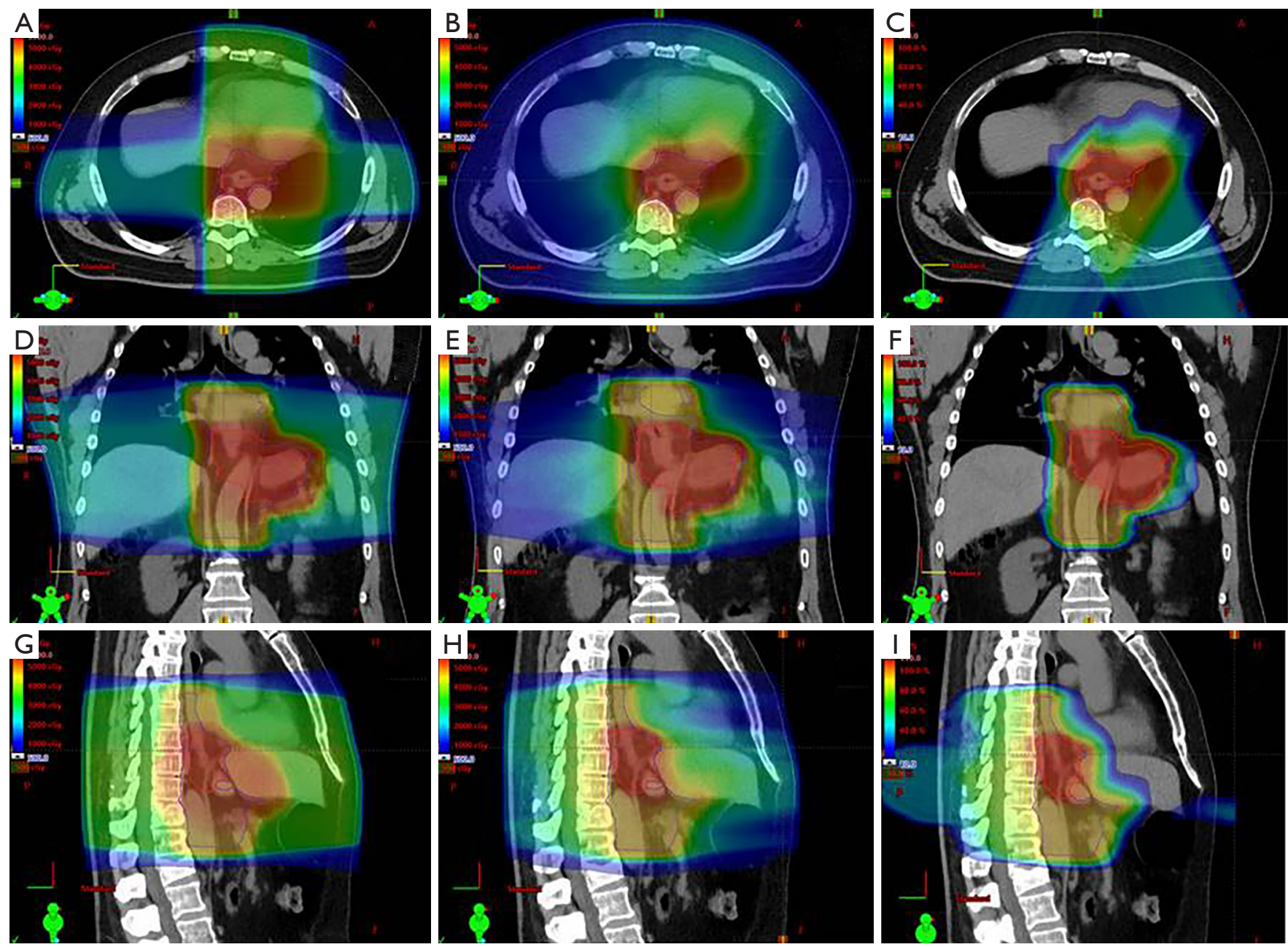

Figure 2 Three-Dimensional conformal (3D-CRT), intensity modulated radiotherapy (IMRT), and pencil beam scanning proton beam radiotherapy (PBT) plans for a patient with locally advanced gastroesophageal junction adenocarcinoma treated with 50 Gy in 25 fractions. Panels A-C show axial slices of 3DCRT (A), IMRT (B), and PBT (C); panels D-F show coronal slices of 3DCRT (D), IMRT (E), and PBT (F); panels G-I show sagittal slices of 3DCRT (G), IMRT (H), and PBT (I). Red and orange indicate higher doses (45-50 Gy), green indicates medium doses (30-40 Gy), and blue indicates lower doses (5-20 Gy). Note sparing of the heart and lungs with IMRT vs. 3DCRT, and PBT vs. IMRT.

of beam angles is important to minimize WET changes over the breathing cycles (26). Periodic verification scans, which are CT scans in the treatment position, are needed to monitor the integrity of the treatment plan over the course of the treatment. The frequency of verification scans vary by institutional preference and the availability of on-board image guidance. Adaptive replanning may be needed if anatomic changes lead to deficiencies in the dose distribution.

\section{Clinical outcomes of PBT}

Sugahara et al. published an early experience from Japan of 46 patients with locoregionally confined disease which suggested the feasibility, safety, and efficacy of PBT for the treatment of esophagus cancer (27). The majority of patients in this study were treated with a combination of PBT and photon radiotherapy (XRT), although six patients were treated with PBT alone. A more recent series from the same institution reported outcomes of definitive PBT (60-70 Gy) with concurrent chemotherapy for patients with stage I-III esophageal cancer. There were no observed cases of grade 3 (severe or medically significant) or higher cardiopulmonary toxicity. The 2-year rates of local-regional control and disease-specific survival were $66 \%$ and $77 \%$, 
respectively, consistent with previously reported series of patients treated with XRT (28). Both of these studies were limited almost exclusively to patients with squamous cell carcinoma.

Lin et al. reported MD Anderson Cancer Center's preliminary experience of treating 62 patients with esophageal cancer with concurrent chemotherapy and PSPT (29). Reflecting a typical Western patient population, approximately $75 \%$ of patients had adenocarcinoma. Patients were treated with a median dose of 50.4 Gy. Most patients experienced grade 1-2 (asymptomatic, mild, or moderate) toxicities, but there were few severe toxicities. In patients undergoing subsequent surgery (47\%), pathologic complete response was observed in $28 \%$ and up to $50 \%$ when including near pathologic complete response. Three-year OS was 52\%. More recently, MD Anderson Cancer Center published their early experience utilizing concurrent chemotherapy and IMPT for the treatment of esophagus cancer in 19 patients (30). After a median dose of 50.4 Gy, clinical complete response was observed in $84 \%$. The most common acute grade 2 and 3 toxicities were esophagitis ( $\mathrm{N}=9$ and $\mathrm{N}=3$, respectively) and fatigue ( $\mathrm{N}=5$ and $\mathrm{N}=3$, respectively). Late grade 2 and 3 toxicities were rare. There were no non-hematologic grade 4 or 5 (life-threatening consequences or death related to adverse event) toxicities. One year OS was $100 \%$.

Recent retrospective series and prospective studies have compared outcomes of patients treated with PBT with patients treated with photon techniques (3D or IMRT). While it has not been definitively clarified if the dosimetric advantages of PBT translate to improved long term outcomes, these available studies have assessed endpoints of oncologic outcomes, toxicities, patient-reported quality of life during CRT, postoperative morbidity (in trimodality patients), and lymphopenia during CRT.

A retrospective study performed at MD Anderson Cancer Center did describe possible OS and oncologic outcome benefits in patients treated with PBT for esophageal cancer as compared to those treated with IMRT (31). This study included 343 patients, with 132 and 211 receiving PBT and IMRT, respectively. At 5 years, the PBT group was found to have significantly higher OS ( $42 \%$ vs. $32 \% ; \mathrm{P}=0.001)$, progression-free survival ( $35 \%$ vs. $20 \%$; $\mathrm{P}=0.001$ ), and distant metastasis-free survival (65\% vs. 50\%; $\mathrm{P}=0.031)$ (31). Although these are promising results, prospective studies are necessary for confirmation.

In an effort to provide prospective data to establish improved clinical outcomes by utilizing PBT, MD Anderson Cancer Center recently published results of a randomized phase IIB trial, assigning 145 patients to PBT or IMRT for the neoadjuvant or definitive treatment of locally advanced esophagus cancer (32). Of the patients assigned to PBT, $80 \%$ received PSPT, with the remainder receiving IMPT. Total toxicity burden, a measure of the cumulative severity of multiple adverse events that patients with esophagus cancer may experience after CRT with or without surgery, was measured up to 12 months after randomization. The mean total toxicity burden was found to be 2.3 times higher for patients treated with IMRT than those treated with PBT, providing further data that PBT may reduce the risk and severity of adverse events in patients treated with RT for esophagus cancer. The 3-year progression-free survival and OS were similar in both cohorts.

Patient reported outcomes (PROs) have been utilized by Mayo Clinic as a tool to demonstrate that the dosimetric advantages achieved by using PBT for the neoadjuvant or definitive treatment of esophagus cancer are clinically meaningful (33). This study analyzed changes of prospectively collected Functional Assessment of Cancer Therapy-Esophagus (FACT-E) questionnaires conducted prior to and in the final week of chemoradiation. All proton patients were treated with PBS, and photon patients were treated with either 3D (30\%) or IMRT $(70 \%)$ techniques. The mean decline in observed FACT-E scores was less for PBT compared to photons $(-12.7$ vs. $-20.6 ; \mathrm{P}=0.026)$, suggesting patients treated with $\mathrm{PBT}$ may experience less acute toxicity (33).

Surgical complications after neoadjuvant CRT combined with surgical resection are common in esophagus cancer. Many of these complications are cardiopulmonary in nature. Wang et al. conducted a study investigating factors associated with postoperative complications in a cohort of patients who received neoadjuvant chemoradiation (9). Treatment with 3D-CRT versus IMRT or PBT was associated with increased complications. Mean lung dose was also strongly associated with pulmonary complications, and lung dose trended lower in patients treated with PBT. Another study compared postoperative outcomes of patients with esophageal cancer treated with trimodality therapy at three major academic centers (34). Patients treated with PBT had significantly shorter hospital stays after esophagectomy, compared with 3D-CRT and IMRT. The mean length of stay was 13.2 days, 11.6 days, and 9.3 days for 3D-CRT, IMRT, and PBT, respectively $(\mathrm{P}<0.001)$.

Grade 4 lymphopenia during radiotherapy has been associated with higher rates of distant metastasis and decreased OS in esophagus cancer (35). A study by MD 
Anderson Cancer Center reported a greater proportion of patients developed grade 4 lymphopenia after IMRT compared to $\mathrm{PBT}(40 \%$ vs. $18 \% ; \mathrm{P}<0.0001)$, which remained significant on multivariable analysis (36). These findings were corroborated in a study conducted at Mayo Clinic investigating the effect of concurrent chemoradiation using 3D-CRT, IMRT, and IMPT (37). Patients receiving radiotherapy with either $3 \mathrm{D}-\mathrm{CRT}$ or IMRT had significantly higher rates of grade 4 lymphopenia compared to those treated with $\mathrm{PBT}(56 \%$ vs. $22 \% ; \mathrm{P}<0.01)$, which remained statistically significant in a propensity-score matched cohort ( $60 \%$ vs. $24 \%$; $\mathrm{P}<0.001)$. While the impact of radiotherapy-related lymphopenia on response rates to immunotherapy remains unclear, one could hypothesize that the preservation of lymphocyte counts with PBT may improve the efficacy of immunotherapy, and thus support the design of clinical trials within this space.

\section{Re-irradiation feasibility}

Re-irradiation for local recurrences or metachronous primaries is a technically challenging task that carries a significant risk of complications. The radiation exposure to the heart, lungs, and spinal cord approaches the maximal safe limits when utilizing radiotherapy for the neoadjuvant or definitive treatment of esophagus cancer. In the setting of prior thoracic radiation, the therapeutic ratio of radiotherapy for a metachronous or locally recurrent esophagus cancer is low, as there is a high risk of damaging nearby normal organs that have already been exposed to radiation. It is imperative to minimize doses to OARs in the re-irradiation setting. Exploiting the physical properties of PBT may increase the therapeutic ratio of re-irradiation for esophagus cancer.

Fernandes et al. reported 14 patients with a history of thoracic radiation and newly diagnosed or locally recurrent esophagus cancer, treated with PBT in an effort to demonstrate feasibility and acceptable rates of acute toxicity (38). After a median interval of 32 months from prior irradiation, patients received a median re-irradiation dose of 54 Gy with a median cumulative prescription dose of 109.8 Gy. Five patients experienced acute grade 3+ toxicity, including grade 3 dysphagia, dehydration, and pneumonia, and 1 patient experienced a grade 5 esophagopleural fistula, thought to be secondary to tumor progression. Four patients experienced grade 3 late toxicities, which included heart failure, esophageal stenosis, esophageal ulceration from tumor progression, and percutaneous endoscopic gastrostomy tube dependence. One patient experienced a late grade 5 toxicity of esophageal ulcer, thought to be related to tumor progression. These data suggest that PBT reirradiation can be delivered without meeting dose limiting cardiopulmonary toxicity; however, local in-field toxicity continues to be a major challenge, leading to significant acute and late toxicities. For a highly selected subgroup with marginal or out of field recurrences, PBT may increase the therapeutic ratio by minimizing dose to the lungs and heart in the re-irradiation setting.

\section{Future directions}

Although PBT seems promising for the treatment of esophagus cancer, a phase III randomized trial that demonstrates meaningful clinical benefits in either reducing toxicity or improving oncologic outcomes has not been completed. NRG-GI006 is currently accruing with a goal of answering this question. This trial includes patients with stage I-IVA adenocarcinoma or squamous cell carcinoma of the esophagus, receiving $50.4 \mathrm{~Gy}$ in 28 fractions with concurrent paclitaxel and carboplatin. Study participants will be randomized 1:1 between radiotherapy with PBT versus IMRT. Appropriate patients may undergo an esophagectomy within 4-8 weeks after completion of chemoradiotherapy. The co-primary objectives are to determine if OS is improved or non-inferior with PBT compared to IMRT and if there are less grade 3+ cardiopulmonary adverse effects with PBT than with IMRT (39).

Although dose escalation for esophagus cancer did not increase survival or locoregional control on the INT 0123 phase III trials using photon therapy (40), dose escalation with proton therapy has not been evaluated in a phase III trial. University of Pennsylvania is currently enrolling patients in a phase I clinical trial aiming to identify the maximally tolerated radiation dose of dose-escalated proton radiotherapy in combination with carboplatin/paclitaxel in the preoperative setting for esophagus cancer as well as estimate pathologic response rates after esophagectomy with escalated doses of radiotherapy (41).

Recent investigations have shown that radiotherapy of tumor cells leads to the release of tumor-associated antigens into the tumor microenvironment, which may facilitate cytotoxic T-cell destruction of tumor cells (42-44). It has also been postulated that PBT, with its higher linear energy transfer and relative biologic effectiveness may further potentiate the radiotherapy effect in the tumor microenvironment, as well as minimize dose to surrounding 
non-target structures, including lymph nodes and blood, which are essential for optimal immune response. It has been demonstrated that PBT leads to less grade 4 lymphopenia than XRT, which may lead to a more robust immune response $(37,45)$. Several single- and multi-institution trials are currently investigating the role of immunotherapy in the treatment of localized esophagus cancer in hopes the addition of immunotherapy may lead to improved oncologic outcomes (46-48). Utilizing PBT may further increase the benefit of immunotherapy by stimulating the immune system through release of tumor-associated antigens and modifying the tumor microenvironment.

\section{Conclusions}

There is a growing body of evidence suggesting potential clinical advantages of PBT in the treatment of esophagus cancer. PBT may provide an advantage to safely intensifying chemoradiotherapy or to combining immunotherapy with radiotherapy. Currently accruing clinical trials will likely provide more insight into the future of esophageal PBT.

\section{Acknowledgments}

Funding: None.

\section{Footnote}

Provenance and Peer Review: This article was commissioned by the Guest Editor (Chi Wan Koo) for the series "Contemporary Practice in Thoracic Neoplasm Diagnosis, Evaluation and Treatment" published in Fournal of Thoracic Disease. The article was sent for external peer review organized by the Guest Editor and the editorial office.

Conflicts of Interest: All authors have completed the ICMJE uniform disclosure form (available at: http://dx.doi. org/10.21037/jtd-2019-cptn-06). The series "Contemporary Practice in Thoracic Neoplasm Diagnosis, Evaluation and Treatment" was commissioned by the editorial office without any funding or sponsorship. The authors have no other conflicts of interest to declare.

Ethical Statement: The authors are accountable for all aspects of the work in ensuring that questions related to the accuracy or integrity of any part of the work are appropriately investigated and resolved.
Open Access Statement: This is an Open Access article distributed in accordance with the Creative Commons Attribution-NonCommercial-NoDerivs 4.0 International License (CC BY-NC-ND 4.0), which permits the noncommercial replication and distribution of the article with the strict proviso that no changes or edits are made and the original work is properly cited (including links to both the formal publication through the relevant DOI and the license). See: https://creativecommons.org/licenses/by-nc-nd/4.0/.

\section{References}

1. Cancer Statistics Center - Key Statistics for Esophageal Cancer. Accessed April 17, 2020. Available online: https:// www.cancer.org/cancer/esophagus-cancer/about/keystatistics.html

2. SEER Cancer Stat Facts: Esophageal Cancer. National Cancer Institute, Bethesda, MD. Available online: https:// seer.cancer.gov/statfacts/html/esoph.html

3. Shapiro J, van Lanschot JJB, Hulshof M, et al. Neoadjuvant chemoradiotherapy plus surgery versus surgery alone for oesophageal or junctional cancer (CROSS): long-term results of a randomised controlled trial. Lancet Oncol 2015;16:1090-8.

4. Morota M, Gomi K, Kozuka T, et al. Late toxicity after definitive concurrent chemoradiotherapy for thoracic esophageal carcinoma. Int J Radiat Oncol Biol Phys 2009;75:122-8.

5. Ishikura S, Nihei K, Ohtsu A, et al. Long-term toxicity after definitive chemoradiotherapy for squamous cell carcinoma of the thoracic esophagus. J Clin Oncol 2003;21:2697-702.

6. Gayed I, Gohar S, Liao Z, et al. The clinical implications of myocardial perfusion abnormalities in patients with esophageal or lung cancer after chemoradiation therapy. Int J Cardiovasc Imaging 2009;25:487-95.

7. Beukema JC, van Luijk P, Widder J, et al. Is cardiac toxicity a relevant issue in the radiation treatment of esophageal cancer? Radiother Oncol 2015;114:85-90.

8. Thrift AP. The epidemic of oesophageal carcinoma: Where are we now? Cancer Epidemiol 2016;41:88-95.

9. Wang J, Wei C, Tucker SL, et al. Predictors of postoperative complications after trimodality therapy for esophageal cancer. Int J Radiat Oncol Biol Phys 2013;86:885-91.

10. Wang SL, Liao Z, Vaporciyan AA, et al. Investigation of clinical and dosimetric factors associated with postoperative 
pulmonary complications in esophageal cancer patients treated with concurrent chemoradiotherapy followed by surgery. Int J Radiat Oncol Biol Phys 2006;64:692-9.

11. Hatakenaka M, Yonezawa M, Nonoshita T, et al. Acute cardiac impairment associated with concurrent chemoradiotherapy for esophageal cancer: magnetic resonance evaluation. Int J Radiat Oncol Biol Phys 2012;83:e67-73.

12. Bradley JD, Paulus R, Komaki R, et al. Standarddose versus high-dose conformal radiotherapy with concurrent and consolidation carboplatin plus paclitaxel with or without cetuximab for patients with stage IIIA or IIIB non-small-cell lung cancer (RTOG 0617): a randomised, two-by-two factorial phase 3 study. Lancet Oncol 2015;16:187-99.

13. Darby SC, Ewertz M, McGale P, et al. Risk of ischemic heart disease in women after radiotherapy for breast cancer. N Engl J Med 2013;368:987-98.

14. Deng JY, Wang C, Shi XH, et al. Reduced toxicity with three-dimensional conformal radiotherapy or intensitymodulated radiotherapy compared with conventional two-dimensional radiotherapy for esophageal squamous cell carcinoma: a secondary analysis of data from four prospective clinical trials. Dis Esophagus 2016;29:1121 7.

15. Tao H, Zhou Y, Yao C, et al. Phase II Trial of IntensityModulated Radiotherapy Concurrent With Chemotherapy for Postoperative Node-Positive Esophageal Squamous Cell Carcinoma. Oncol Res 2017;25:1357-62.

16. Lin SH, Wang L, Myles B, et al. Propensity score-based comparison of long-term outcomes with 3-dimensional conformal radiotherapy vs intensity-modulated radiotherapy for esophageal cancer. Int J Radiat Oncol Biol Phys 2012;84:1078-85.

17. Lin SH, Zhang N, Godby J, et al. Radiation modality use and cardiopulmonary mortality risk in elderly patients with esophageal cancer. Cancer 2016;122:917-28.

18. McDermott PM, Orton, Colin G. The Physics \& Technology of Radiation Therapy. Madison, Wisconsin: Medical Physics Publishing, 2010.

19. Mitin T, Zietman AL. Promise and pitfalls of heavyparticle therapy. J Clin Oncol 2014;32:2855-63.

20. Shiraishi Y, Xu C, Yang J, et al. Dosimetric comparison to the heart and cardiac substructure in a large cohort of esophageal cancer patients treated with proton beam therapy or Intensity-modulated radiation therapy. Radiother Oncol 2017;125:48-54.

21. Zhang X, Zhao K-1, Guerrero TM, et al. Four-dimensional computed tomography-based treatment planning for intensity-modulated radiation therapy and proton therapy for distal esophageal cancer. Int J Radiat Oncol Biol Phys 2008;72:278-87.

22. Welsh J, Gomez D, Palmer MB, et al. Intensity-modulated proton therapy further reduces normal tissue exposure during definitive therapy for locally advanced distal esophageal tumors: a dosimetric study. Int J Radiat Oncol Biol Phys 2011;81:1336-42.

23. Tryggestad EJ, Liu W, Pepin MD, et al. Managing treatment-related uncertainties in proton beam radiotherapy for gastrointestinal cancers. J Gastrointest Oncol 2020;11:212-24.

24. Chuong MD, Hallemeier CL, Jabbour SK, et al. Improving Outcomes for Esophageal Cancer using Proton Beam Therapy. Int J Radiat Oncol Biol Phys 2016;95:488-97.

25. Yaremko BP, Guerrero TM, McAleer MF, et al. Determination of respiratory motion for distal esophagus cancer using four-dimensional computed tomography. Int J Radiat Oncol Biol Phys 2008;70:145-53.

26. Yu J, Zhang X, Liao L, et al. Motion-robust intensitymodulated proton therapy for distal esophageal cancer. Med Phys 2016;43:1111-8.

27. Sugahara S, Tokuuye K, Okumura T, et al. Clinical results of proton beam therapy for cancer of the esophagus. Int J Radiat Oncol Biol Phys 2005;61:76-84.

28. Ishikawa H, Hashimoto T, Moriwaki T, et al. Proton beam therapy combined with concurrent chemotherapy for esophageal cancer. Anticancer Res 2015;35:1757-62.

29. Lin SH, Komaki R, Liao Z, et al. Proton beam therapy and concurrent chemotherapy for esophageal cancer. Int J Radiat Oncol Biol Phys 2012;83:e345-51.

30. Prayongrat A, Xu C, Li H, et al. Clinical outcomes of intensity modulated proton therapy and concurrent chemotherapy in esophageal carcinoma: a single institutional experience. Adv Radiat Oncol 2017;2:301-7.

31. Xi M, Xu C, Liao Z, et al. Comparative Outcomes After Definitive Chemoradiotherapy Using Proton Beam Therapy Versus Intensity Modulated Radiation Therapy for Esophageal Cancer: A Retrospective, SingleInstitutional Analysis. Int J Radiat Oncol Biol Phys 2017;99:667-76.

32. Lin SH, Hobbs BP, Verma V, et al. Randomized Phase IIB Trial of Proton Beam Therapy Versus Intensity-Modulated Radiation Therapy for Locally Advanced Esophageal Cancer. J Clin Oncol 2020;38:1569-79.

33. Garant A, Whitaker TJ, Spears GM, et al. A Comparison of Patient-Reported Health-Related Quality of Life 
During Proton Versus Photon Chemoradiation Therapy for Esophageal Cancer. Pract Radiat Oncol 2019;9:410-7.

34. Lin SH, Merrell KW, Shen J, et al. Multi-institutional analysis of radiation modality use and postoperative outcomes of neoadjuvant chemoradiation for esophageal cancer. Radiother Oncol 2017;123:376-81.

35. Davuluri R, Jiang W, Fang P, et al. Lymphocyte Nadir and Esophageal Cancer Survival Outcomes After Chemoradiation Therapy. Int J Radiat Oncol Biol Phys 2017;99:128-35.

36. Shiraishi Y, Fang P, Xu C, et al. Severe lymphopenia during neoadjuvant chemoradiation for esophageal cancer: A propensity matched analysis of the relative risk of proton versus photon-based radiation therapy. Radiother Oncol 2018;128:154-60.

37. Routman DM, Garant A, Lester SC, et al. A Comparison of Grade 4 Lymphopenia With Proton Versus Photon Radiation Therapy for Esophageal Cancer. Adv Radiat Oncol 2019;4:63-9.

38. Fernandes A, Berman AT, Mick R, et al. A Prospective Study of Proton Beam Reirradiation for Esophageal Cancer. Int J Radiat Oncol Biol Phys 2016;95:483-7.

39. NRG-GI006, Phase III Randomized Trial of Proton Beam Therapy (PBT) versus Intensity Modulated Photon Radiotherapy (IMRT) for the Treatment of Esophageal Cancer.

40. Minsky BD, Pajak TF, Ginsberg RJ, et al. INT 0123 (Radiation Therapy Oncology Group 94-05) phase III trial of combined-modality therapy for esophageal cancer: high-dose versus standard-dose radiation therapy. J Clin Oncol 2002;20:1167-74.

Cite this article as: Gergelis KR, Jethwa KR, Tryggestad EJ, Ashman JB, Haddock MG, Hallemeier CL. Proton beam radiotherapy for esophagus cancer: state of the art. J Thorac Dis 2020;12(11):7002-7010. doi: 10.21037/jtd-2019-cptn-06
41. ClinicalTrials.gov. Dose Escalation of Neoadjuvant Proton Beam Radiotherapy With Concurrent Chemotherapy in Locally Advanced Esophageal Cancer. Available online: https://clinicaltrials.gov/ct2/show/NCT02213497

42. Kotera Y, Shimizu K, Mule JJ. Comparative analysis of necrotic and apoptotic tumor cells as a source of antigen(s) in dendritic cell-based immunization. Cancer Res 2001;61:8105-9.

43. Melcher A, Gough M, Todryk S, et al. Apoptosis or necrosis for tumor immunotherapy: what's in a name? J Mol Med (Berl) 1999;77:824-33.

44. Chen Z, Moyana T, Saxena A, et al. Efficient antitumor immunity derived from maturation of dendritic cells that had phagocytosed apoptotic/necrotic tumor cells. Int J Cancer 2001;93:539-48.

45. Lee HJ Jr, Zeng J, Rengan R. Proton beam therapy and immunotherapy: an emerging partnership for immune activation in non-small cell lung cancer. Transl Lung Cancer Res 2018;7:180-8.

46. ClinicalTrials.gov. Association of Radiochemotherapy and Immunotherapy for the Treatment of Unresectable Oesophageal caNcer (ARION). Available online: https:// clinicaltrials.gov/ct2/show/NCT03777813

47. ClinicalTrials.gov. Combination of Chemoradiation With Immunotherapy in Inoperable œesophageal Cancer (CRUCIAL). Available online: https://clinicaltrials.gov/ ct2/show/NCT03437200

48. ClinicalTrials.gov. Safety and Feasibility of Irradiation and Nivolumab in Esophageal Cancer (INEC). Available online: https://clinicaltrials.gov/ct2/show/NCT03544736 